\title{
Estudo da interferência de óleos essenciais sobre a atividade de alguns antibióticos usados na clínica
}

\author{
Rinalda A. Guerra de Oliveira, Edeltrudes O. Lima*, Wellington L. Vieira, Kristerson R. Luna \\ Freire, Vinicius N. Trajano, Igara O. Lima, Evandro L. Souza, \\ Manuella S. Toledo, Raimundo N. Silva-Filho
}

\author{
Laboratório de Micologia, Departamento de Ciências Farmacêuticas, Centro de Ciências da Saúde, Universidade \\ Federal da Paraíba, Cidade Universitária, 58051-970, João Pessoa, PB, Brasil
}

\begin{abstract}
RESUMO: O objetivo deste estudo foi investigar a interferência dos óleos essenciais de Lippia sidoides Cham, Plectranthus amboinicus Lour Spr., Conyza bonariensis L. e Eucalyptus citriodora Hook sobre o efeito de antibióticos utilizados na clínica. Os ensaios foram realizados com ampicilina $(10 \mu \mathrm{g} / \mathrm{mL})$, cefalotina $(30 \mu \mathrm{g} / \mathrm{mL})$, cloranfenicol $(30 \mu \mathrm{g} / \mathrm{mL})$, gentamicina $(10 \mu \mathrm{g} / \mathrm{mL})$ e tetraciclina $(30 \mu \mathrm{g} / \mathrm{mL})$ isolados e em associação com os óleos essenciais $(4 \% \mathrm{v} / \mathrm{v})$ através do método de difusão em meio sólido utilizando discos de papel de filtro. Os resultados mostraram interferência de alguns óleos essenciais sobre a atividade dos antibióticos ensaiados. Observou-se o desenvolvimento de halos de inibição do crescimento bacteriano com diferentes diâmetros quando da aplicação de antibióticos isolados e em combinação com os óleos essenciais. A ocorrência de comportamento sinérgico ou antagônico foi observada mais proeminentemente nas interações com cepas de S. aureus e S. epidermidis. De outra forma, observou-se uma menor interferência dos óleos essenciais no efeito dos antibióticos sobre as cepas Gram negativas, principalmente $P$. aeruginosa. Estes resultados mostram que o uso de produtos derivados de plantas pode em algumas situações interferir sobre a efetividade de antibióticos de uso no tratamento clínico.
\end{abstract}

Unitermos: Plantas medicinais, óleos essenciais, antibióticos, uso associado, interferência.

\begin{abstract}
Study of the interference of essential oils on the activity of some antibiotic used clinically". The aim of this study was to evaluate the interference of essential oils from Lippia sidoides Cham, Plectranthus amboinicus Lour Spr., Conyza bonariensis L. and Eucalyptus citriodora Hook on the antibacterial effect of clinically used antibiotics. The assays were carried out with ampicillin $(10 \mu \mathrm{g} / \mathrm{mL})$, cephalotin $(30 \mu \mathrm{g} / \mathrm{mL})$, chloraphenicol $(30 \mu \mathrm{g} / \mathrm{mL})$, gentamicin $(10 \mu \mathrm{g} / \mathrm{mL})$ and tetracyclin $(30 \mu \mathrm{g} / \mathrm{mL})$ isolated and associated with essential oils $(4 \% \mathrm{v} / \mathrm{v})$ by the solid medium diffusion procedure using filter paper discs. The results showed interference of some essential oils on the antibacterial activity of the assayed antibiotics. It was observed the development of growth inhibition halo with different diameters when the antibiotics were applied alone and associated with essential oils. Synergic or antagonic behavior was more prominent in interactions with $S$. aureus and S. epidermidis strains. On the other hand, it was observed a smaller interference of essential oils on the effect of the antibiotics against Gram negative strains, mainly $P$. aeruginosa. These results show that the use of plants by-products could some times interfere on the effectiveness of antibiotics used clinically.
\end{abstract}

Keywords: Medicinal plants, essential oils, antibiotics, associated use, interference.

\section{INTRODUÇÃO}

As plantas com propriedades terapêuticas utilizadas no cuidado de saúde tradicional constituem uma importante fonte de novos compostos biologicamente ativos. Elas aparecem como parte do cuidado tradicional de saúde em muitas partes do mundo ao longo de décadas e têm despertado o interesse de vários pesquisadores (Ceballos et al., 1993; Lima, 1996; Cunha, 1995; Cowan, 1999; Farias; Lima, 2000; Belém, 2002; Michelin et al., 2005). Na medicina popular, as plantas são utilizadas concomitantemente ao uso de medicamentos convencionais (Amorim, 1999). Neste uso associado, as plantas medicinais e/ou seus subprodutos podem atuar inibindo ou intensificando o efeito terapêutico dos medicamentos convencionais, bem como não interferir na resposta esperada (Nascimento et al., 2000). Tal uso associativo na dermatologia, plantas ou seus subprodutos junto ao uso de medicamentos convencionais, colocam o paciente muitas vezes em risco, já que pode desencadear fitodermatoses decorrente de mecanismo de irritação ou fotossensibilidade. Ainda, tal prática pode dificultar o diagnóstico clínico ao considerar que o próprio usuário não avalia a importância do repasse da informação ao serviço de saúde no momento do atendimento.

Os óleos essenciais são originados do 
metabolismo secundário das plantas e possuem composição química complexa, destacando-se a presença de terpenos e fenilpropanoides (Gonçalves et al., 2003; Silva et al., 2003). Os óleos essenciais constituem os elementos voláteis contidos em muitos órgãos vegetais, e, estão relacionados com diversas funções necessárias à sobrevivência vegetal, exercendo papel fundamental na defesa contra microrganismos (Siqui et al., 2000). Ainda, tem sido estabelecido cientificamente que cerca de $60 \%$ dos óleos essenciais possuem propriedades antifúngicas e $35 \%$ exibem propriedades antibacterianas (Bhavanani; Ballow, 1992).

Nesse contexto foi estudada a interferência in vitro de óleos essenciais de plantas utilizadas na medicina popular no tratamento de doenças dermatológicas infecciosas sobre a atividade de antibióticos utilizados na clínica.

\section{MATERIAL E MÉTODOS}

\section{Material botânico}

As plantas selecionadas para o estudo estão listadas na Tabela 1. Tomou-se como critério de seleção o seu uso popular, o estudo etnobotânico, a atividade antimicrobiana detectada em estudos anteriores e experiências clínicas preliminares (Lima, 1996; Amorim, 1999; Belém, 2002). A coleta das plantas foi realizada no horto do Laboratório de Tecnologia Farmacêutica (LTF) da Universidade Federal da Paraíba (UFPB), pelo agrônomo Fernando Antônio Cavalcante Viana. A espécie Plectranthus amboinicus Lour Spr., Lamiaceae, acompanhada da respectiva exsicata foi encaminhada em setembro de 2002 para o Herbário IPA - Dárdano de Andrade Lima, Empresa Pernambucana de Pesquisa Agropecuária, sendo determinada pela botânica $\mathrm{Dr}^{\mathrm{a}}$ Rita de Cássia A. Pereira. As demais espécies foram determinadas pelo botânico José Roberto Leite do Departamento de Sistemática e Ecologia/Centro de Ciências Exatas e da Natureza/UFPB, catalogadas e registradas no Herbário Lauro Pires Xavier JPB (Pontes, 2002).

Os óleos essenciais foram fornecidos pelo Laboratório de Tecnologia Farmacêutica, Centro de Ciências da Saúde, UFPB, sendo extraídos conforme procedimento padrão (Craveiro et al., 1981; Matos, 1988).

\section{Microrganismos}

Foram utilizadas como microrganismos testes cepas de Staphylococcus aureus (ATCC-6538), S. epidermidis (ATCC-12228), Escherichia coli (LMB-4) e Pseudomonas aeruginosa (LMB-6). Foram preparadas suspensões das cepas testes em solução salina $(\mathrm{NaCl}$ a $0,85 \% \mathrm{p} / \mathrm{v}$ ), as quais foram padronizadas de acordo com o tubo 0,5 da escala McFarland correspondendo à concentração de aproximadamente $10^{8}$ Unidades
Formadoras de Colônia - (UFC/mL).

\section{Antibióticos}

Ampicilina $(10 \mu \mathrm{g} / \mathrm{mL})$, cefalotina $(10 \mu \mathrm{g} / \mathrm{mL})$, cloranfenicol $(30 \mu \mathrm{g} / \mathrm{mL})$, gentamicina $(10 \mu \mathrm{g} / \mathrm{mL})$ e tetraciclina $(30 \mu \mathrm{g} / \mathrm{mL})$ foram selecionados para inclusão no estudo tomando como base sua disponibilidade na rede básica de saúde. O estudo de observação de sensibilidade das cepas bacterianas frente aos antibióticos foi realizado através da técnica de difusão em meio sólido utilizandose discos de papel de filtro (Sensibiodisc-CECON) (Bauer et al.,1966).

\section{Preparação das soluções dos óleos essenciais}

Em tubo estéril foi adicionado $0,4 \mathrm{~mL}$ do óleo essencial, $0,04 \mathrm{~mL}$ de Tween 80 e q.s.p. $5 \mathrm{~mL}$ de água destilada estéril, sendo agitado por 5 minutos usando-se aparelho Vortex (Fanem), obtendo-se uma solução com concentração de $8 \%$ do óleo essencial. A partir de tal solução, utilizando o procedimento de diluição seriada, obteve-se a solução com concentração correspondente a Concentração Inibitória Mínima-CIM dos óleos essenciais ensaiados (Allegrini et al., 1973).

\section{Estudo da interferência dos óleos essenciais sobre o efeito de antibióticos}

O estudo da interferência dos óleos essenciais sobre a efetividade dos antibióticos foi realizado através da técnica de difusão em meio sólido utilizando discos de papel de filtro. Os óleos essenciais foram estudados na concentração de 4\%, correspondendo a CIM determinada em pesquisas anteriores (Ceballos et al., 1993; Lima et al., 2003; Araújo et al., 2004; Nogueira, 2004), incluindo os mesmos óleos essenciais e microrganismos ensaiados neste estudo, realizados pelo grupo de pesquisa do Laboratório de Micologia, Departamento de Ciências Farmacêuticas, Centro de Ciências da Saúde, UFPB.

Os discos contendo os antibióticos nas suas respectivas concentrações foram embebidos com $20 \mu \mathrm{L}$ da CIM do óleo essencial, sendo em seguida colocados em placas de Petri estéreis contendo ágar Muller-Hinton inoculado com $1 \mathrm{~mL}$ das suspensões bacterianas. Após incubação das placas a $37^{\circ} \mathrm{C}$ por 48 horas, foi observada a interferência da CIM dos óleos essenciais sobre o efeito dos antibióticos sobre as cepas bacterianas ensaiadas. Considerou-se como efeito sinergístico, quando da ocorrência de halo de inibição do crescimento microbiano formado pela aplicação combinada do óleo essencial (OE) mais o antibiótico (AB) com diâmetro $\geq$ que $2 \mathrm{~mm}$, quando comparado com o halo de inibição formado pela ação do $\mathrm{AB}$ isoladamente. Quando da formação de halo de inibição decorrente da ação combinada $\mathrm{AB}$ e $\mathrm{OE}$ de menor diâmetro daquele desenvolvido pela ação isolada do $\mathrm{AB}$ considerou-se efeito antagônico. Foi considerado 
Tabela 1. Plantas medicinais selecionadas para o estudo da interferência de seus óleos essenciais sobre o efeito antibacteriano de alguns antibióticos.

\begin{tabular}{|c|c|c|c|c|}
\hline Espécie/ Família & Nome Vulgar & Indicação Popular & $\begin{array}{c}\text { Parte da Planta } \\
\text { Usada }\end{array}$ & $\begin{array}{c}\mathrm{N}^{\circ} \mathrm{da} \\
\text { Exsicata }\end{array}$ \\
\hline $\begin{array}{l}\text { Conyza bonariensis L. } \\
\text { (Compositae) }\end{array}$ & Rabo de raposa & $\begin{array}{l}\text { Pano branco, coceira, } \\
\text { impingem }\end{array}$ & Folhas & JPB 26391 \\
\hline $\begin{array}{l}\text { Lippia sidoides Cham* } \\
\text { (Verbenaceae) }\end{array}$ & Alecrim pimenta & $\begin{array}{l}\text { Não tem indicação } \\
\text { popular na Paraíba }\end{array}$ & Folhas & JPB 26366 \\
\hline $\begin{array}{l}\text { Plectrantus amboinicus } \\
\text { Lour Spr. (Lamiaceae) }\end{array}$ & $\begin{array}{l}\text { Hortelã da folha } \\
\text { grossa }\end{array}$ & $\begin{array}{l}\text { Impingem, coceira, feridas } \\
\text { da pele, expectorante }\end{array}$ & Folhas & IPA 65501 \\
\hline $\begin{array}{l}\text { Eucalypthus citriodora } \\
\text { Hook (Myrtaceae) }\end{array}$ & Eucalipto & Feridas da pele & Folhas & JPB 26503 \\
\hline
\end{tabular}

* espécie recomendada pelo Programa Farmácias vivas (Matos, 1994).

Tabela 2. Sensibilidade de cepas bacterianas frente à ação de diferentes antibióticos (resultados expressos em diâmetro dos halos de inibição do crescimento microbiano).

\begin{tabular}{lccccc}
\hline \multirow{2}{*}{ Microrganismos } & \multicolumn{5}{c}{ Antibióticos } \\
\cline { 2 - 6 } & $\begin{array}{c}\text { Ampicilina } \\
(10 \mu \mathrm{g} / \mathrm{mL})\end{array}$ & $\begin{array}{c}\text { Cefalotina } \\
(30 \mu \mathrm{g} / \mathrm{mL})\end{array}$ & $\begin{array}{c}\text { Cloranfenicol } \\
(30 \mu \mathrm{g} / \mathrm{mL})\end{array}$ & $\begin{array}{c}\text { Gentamicina } \\
(10 \mu \mathrm{g} / \mathrm{mL})\end{array}$ & $\begin{array}{c}\text { Tetraciclina } \\
(30 \mu \mathrm{g} / \mathrm{mL})\end{array}$ \\
\hline $\begin{array}{l}\text { S. aureus } \\
\text { ATCCC-6538 }\end{array}$ & 18 & 14 & 18 & 25 & 22 \\
$\begin{array}{l}\text { S. epidermidis } \\
\text { ATCC-12228 }\end{array}$ & 0 & 12 & 10 & 25 & 23 \\
$\begin{array}{l}P \text {. aeruginosa } \\
\text { LMB-6 }\end{array}$ & 0 & 0 & 0 & 0 & 15 \\
$\begin{array}{l}\text { E. coli } \\
\text { LMB-4 }\end{array}$ & 0 & 17 & 0 & 22 & 24 \\
\hline
\end{tabular}

como efeito indiferente, quando da observação de halo de inibição conseqüente à aplicação combinada $\mathrm{AB}$ e $\mathrm{OE}$ com diâmetro igual àquele conseqüente da aplicação isolada do AB (Cleeland; Squires, 1991). Todos os ensaios foram realizados em duplicata e os resultados a serem expostos foram obtidos através da média dos resultados dos dois ensaios paralelos.

\section{RESULTADOS E DISCUSSÃO}

Os resultados referentes à sensibilidade das cepas bacterianas frente à ação de antibióticos são mostrados na Tabela 2. Observa-se que as bactérias Gram positivas (S. aureus e S. epidermidis) apresentaram-se como as mais sensíveis, de modo que $S$. aureus foi sensível a todos os antibióticos ensaiados, apresentando halos de inibição do crescimento com diâmetros oscilando entre 13 e $25 \mathrm{~mm}$. S. epidermidis nas interações com cefalotina, cloranfenicol, gentamicina e tetraciclina apresentou halos de inibição do crescimento com diâmetro oscilando entre 10 e $25 \mathrm{~mm}$, embora tenha sido resistente a ampicilina. Entre as cepas Gram negativas, destaca-se a resistência apresentada por $P$. aeruginosa frente aos antibióticos ampicilina, cefalotina, cloranfenicol e gentamicina. E. coli, por sua vez, mostrou-se sensível a cefalotina, gentamicina e tetraciclina.
A Tabela 3 mostra a interferência dos óleos essenciais de plantas medicinais de uso popular sobre a ação antibacteriana de antibióticos utilizados na clínica. Considerando a comparação dos diâmetros dos halos de inibição do crescimento bacteriano nos ensaios com os antibióticos isolados e em associação com os óleos essenciais, pode-se observar, em algumas interações, a ocorrência de interferência dos óleos essenciais sobre o poder antibacteriano dos antibióticos. Esta interferência ocorreu, principalmente, nas interações com as cepas de $S$. aureus e S. epidermidis. E. coli apresentou variados graus de susceptibilidade, ao passo que para $P$. aeruginosa não houve interferência das associações testadas.

Ampicilina, cefalotina e tetraciclina foram os antibióticos que tiveram a maior interferência dos óleos essenciais, sendo observado, na maioria das interações, uma interferência sinérgica. Gentamicina mostrou efeito antagônico na grande maioria das associações. Tal comportamento foi observado, principalmente, quando testado o efeito do uso associativo sobre S. aureus. De uma forma geral, a característica da interferência exercida pelo óleo essencial sobre a ação dos antibióticos variou de acordo com o tipo do antibiótico, tipo do óleo essencial testado em associação, e tipo de cepa bacteriana ensaiada.

O óleo essencial de $L$. sidoides teve uma ação 
Tabela 3. Interferência de óleos essenciais de plantas medicinais de uso popular sobre a ação antibacteriana de antibióticos utilizados na clínica.

\begin{tabular}{|c|c|c|c|c|}
\hline \multirow[b]{2}{*}{ Aplicação combinada } & \multicolumn{4}{|c|}{ Microrganismos } \\
\hline & $\begin{array}{c}\text { S. aureus } \\
\text { ATCC-6538 }\end{array}$ & $\begin{array}{l}\text { S. epidermidis } \\
\text { ATCC- } 12228\end{array}$ & $\begin{array}{l}\text { P. aeruginosa } \\
\text { LMB-6 }\end{array}$ & $\begin{array}{l}\text { E. coli } \\
\text { LMB-4 }\end{array}$ \\
\hline L. sidoides + ampicilina & $20 \uparrow$ & $12 \uparrow$ & $0 *$ & $0 *$ \\
\hline L. sidoides + cefalotina & $20 \uparrow$ & $15 \uparrow$ & $0 *$ & $20 \uparrow$ \\
\hline L. sidoides + cloranfenicol & $18 *$ & $12 \uparrow$ & $0 *$ & $0 *$ \\
\hline L. sidoides + gentamicina & $20 \downarrow$ & $25 *$ & $0 *$ & $22 *$ \\
\hline L. sidoides + tetraciclina & $22 *$ & $20 *$ & $14 \downarrow$ & $23 \downarrow$ \\
\hline P. amboinicus + ampicilina & $20 \uparrow$ & $14 \uparrow$ & $0 *$ & $0 *$ \\
\hline P. amboinicus + cefalotina & $20 \uparrow$ & $15 \uparrow$ & $0 *$ & $19 \uparrow$ \\
\hline P. amboinicus + cloranfenicol & $15 \downarrow$ & $14 \uparrow$ & $0 *$ & $0 *$ \\
\hline P. amboinicus + gentamicina & $20 \downarrow$ & $22 \downarrow$ & $0 *$ & $20 \downarrow$ \\
\hline P. amboinicus + tetraciclina & $23 *$ & $25 \uparrow$ & $14 \downarrow$ & $22 \downarrow$ \\
\hline C. bonariensis + ampicilina & $21 \uparrow$ & $18 \uparrow$ & $0 *$ & $0 *$ \\
\hline C. bonariensis + cefalotina & $23 \uparrow$ & $18 \uparrow$ & $0 *$ & $21 \uparrow$ \\
\hline C. bonariensis + cloranfenicol & $14 \downarrow$ & $13 \uparrow$ & $0 *$ & $0 *$ \\
\hline C. bonariensis + gentamicina & $21 \downarrow$ & $25 *$ & $0 *$ & $21 \downarrow$ \\
\hline C. bonariensis + tetraciclina & $24 \uparrow$ & $25 \uparrow$ & $16 *$ & $25 *$ \\
\hline E. citriodora + ampicilina & $15 \downarrow$ & $11 \uparrow$ & $0 *$ & $0 *$ \\
\hline E. citriodora + cefalotina & $15 *$ & $11 *$ & $0 *$ & $18 *$ \\
\hline E. citriodora + cloranfenicol & $15 \downarrow$ & $13 \uparrow$ & $0 *$ & $0 *$ \\
\hline E. citriodora + gentamicina & $22 \downarrow$ & $23 \downarrow$ & $0 *$ & $20 \downarrow$ \\
\hline E. citriodora + tetraciclina & $25 \uparrow$ & $16 \uparrow$ & $17 \uparrow$ & $26 \uparrow$ \\
\hline
\end{tabular}

a óleos essenciais testados na concentração de 4\%; ampicilina: $10 \mu \mathrm{g} / \mathrm{mL}$; cefalotina: $30 \mu \mathrm{g} / \mathrm{mL}$; cloranfenicol: $30 \mu \mathrm{g} / \mathrm{mL}$; gentamicina: $10 \mu \mathrm{g} / \mathrm{mL}$; tetraciclina: $30 \mu \mathrm{g} / \mathrm{mL}$; $\uparrow$ : efeito sinérgico; $\downarrow$ : efeito antagônico; * efeito indiferente.

potencializadora do efeito antibacteriano de ampicilina, cefalotina e cloranfenicol, embora tal interferência não tenha sido observada nas interações com todos os microrganismos. Destaca-se, o efeito sinérgico exercido pelo óleo essencial de $L$. sidoides sobre cefalotina na interação com $S$. aureus. Nas interações envolvendo as cepas Gram negativas, esse óleo essencial, na maioria das interações, apresentou efeito sinérgico ou antagônico à atividade dos antibióticos. L. sidoides não tem indicação popular como antibacteriano na Paraíba, porém estudos científicos da composição de seu óleo essencial evidenciaram a presença de timol, composto com evidências de ação inibitória contra fungos e bactérias. Sua alcoolatura é indicada para o tratamento de 
micose e escabiose infectada (Matos, 1994, Bara; Vanetti, 1997/1998; Costa et al., 2005).

O óleo essencial de $P$. amboinicus mostrou em algumas interações, um efeito sinérgico sobre a atividade dos antibióticos, sendo que tal efeito foi observado principalmente na interação com S. epidermidis. Estudo realizado por Nogueira (2004) mostrou que o extrato das folhas de P. amboinicus foi ativo sobre cepa de $S$. aures isolada de otite externa. Timol e carvacrol presentes no óleo essencial de P. amboinicus confere-lhe propriedades antibacterianas (Dinizetal., 1997; Matos, 1994). Carvacrol é referido como marcador de poder antimicrobiano de óleos essenciais (Menéndez; González, 1999).

O óleo essencial de C. bonariensis apresentou sinergismo com a maioria dos antibióticos testados. A alcoolatura desta planta tem sido usada topicamente no tratamento de dermatoses, bem como tem sido prescrita pelos médicos do Programa Saúde da Família do Município de João Pessoa/Paraíba (Secretaria de Saúde do Estado da Paraíba, 2002).

O óleo essencial de E. citriodora apresentou sinergismo com ampicilina, cloranfenicol e tetraciclina frente $S$. epidermidis. Com relação a $S$. aureus, houve diminuição da atividade inibitória de ampicilina, cloranfenicol e gentamicina. Tetraciclina, quando aplicado em combinação com o óleo essencial de E. citriodora evidenciou sinergismo nas interações com todas as bactérias testadas. O óleo essencial de E. citriodora é constituído principalmente por citronelal, o qual apresenta propriedade irritante da mucosa do trato respiratório e tem poder anti-séptico ambiental (Simões et al., 1999; Craveiro et al., 1981; Matos, 1994). E. citriodora tem se caracterizado como uma das espécies mais utilizadas no nordeste brasileiro como planta medicinal (Estanislau et al., 2001).

\section{CONCLUSÃO}

De acordo com os resultados obtidos, observa-se que todos os óleos essenciais analisados apresentaram capacidade de exercer uma maior ou menor ação interferente sobre a atividade antibacteriana dos antibióticos ensaiados, seja através de um efeito antagônico ou sinérgico. Esta interferência foi observada principalmente nos ensaios envolvendo as cepas de S. aureus e S. epidermidis. P. aeruginosa foi a cepa menos sensível ao uso combinado dos antibióticos e óleos essenciais. Assim, o uso concomitante de produtos vegetais e medicamentos convencionais merece um olhar muito cuidadoso visto ocorrer à possibilidade de interferir no tratamento de doenças de etiologia bacteriana.

\section{AGRADECIMENTOS}

Ao Prof. Dr. José Maria Barbosa Filho pelos óleos essenciais cedidos. Ao PIBIC pelas bolsas concedidas aos alunos. A dermatologista Dra. Maria de
Fátima de Lacerda Guerra pelas sugestões e apoio. Ao Agrônomo Fernando Antônio Cavalcante Viana pela coleta das plantas. A Dr ${ }^{a}$. Rita de Cássia pela identificação botânica.

\section{REFERÊNCIAS}

Allegrini M, Siméon M, Maillos H, Boiloot A 1973. Èmulsions et applications en microbiologie. Travaux de la Société de Phamacie de Montpellier 33: 73-86.

Amorim JA 1999. Fitoterapia popular e saúde da comunidade: diagnóstico para proposta de integração nos serviços de saúde em Campina Grande, Paraíba. São Paulo, 206p. Tese de Doutorado - Faculdade de Saúde Pública, Universidade de São Paulo.

Araújo JCLV, Lima EO, Ceballos BS, Freire KRL, Souza EL, Santos-Filho L 2004. Ação antimicrobiana de óleos essenciais sobre microrganismos potencialmente causadores de infecções oportunistas. Rev Patol Trop 33: 55-64.

Bara MTF, Vanetti MCD 1997/1998. Estudo da atividade antibacteriana de plantas medicinais, aromáticas e corantes naturais. Rev Bras Farmacogn 7/8: 21-34.

Bawer AW, Kirby WM, Sherris SC, Turck M 1966. Antibiotic susceptibility testing by a standardized single disc method. Am J Clin Pathol 45: 493-496.

Belém LF 2002. Estudo epidemiológico da Pitiríase versicolor no estado da Paraíba e avaliação química $e$ antifúngica de produtos naturais e sintéticos contra seu agente etiológico. João Pessoa, 178p. Tese de Doutorado - Programa de Pós-graduação em Produtos Naturais e Sintéticos Bioativos, Universidade Federal da Paraíba.

Bhavanani SM, Ballow CH 1992. New agents for Gram-positive bacteria. Curr Opin Microbiol 13: 528-534.

Ceballos BSO, Barbosa RCSBC, Lima EO, Urtiga RF 1993. Atividades antimicrobianas de produtos naturais sobre Staphylococcus aureus e Pseudomonas aeruginosa isoladas de águas recreacionais. Rev Bras Farm 74: 4-6.

Cleeland L, Squires E 1991. Evaluation of new antimicrobials in vitro and experimental animal infections. In: Lorian VMD. Antibiotics in Laboratory Medicine. Baltimore: Williams \& Wilkins, p.739-788.

Costa JGM, Rodrigues FFG, Angélico EC, Silva MR, Mota ML, Santos NKA, Cardoso ALH, Lemos TLG 2005. Estudo químico-biológico dos óleos essenciais de Hyptis martiusii, Lippia sidoides e Syzigium aromaticum frente às larvas do Aedes aegypti. Rev Bras Farmacogn 15: 304-309.

Cowan MN 1999. Plant products as antimicrobial agents. Clin Microbiol Rev 12: 564-582.

Craveiro AA, Fernandes AG, Andrade CHS, Matos FJA, Alencar JW, Machado MIL 1981. Óleos essenciais de Plantas do Nordeste. Fortaleza: Editora UFC.

Cunha GMA 1995. Atividade antimicrobiana de plantas popularmente usadas no Ceará. Rev Bras Farm 76: 5-6.

Diniz MFFM, Oliveira RAG, Medeiros ACD, Malta-Júnior RA 1997. Memento fitoterápico: as plantas como alternativa terapêutica; conhecimentos populares e científicos. João Pessoa: Editora Universitária. 
Estanislau AA, Barros FAZ, Peña AP, Santos SC, Ferri PH, Paula JR 2001. Composição química e atividade antibacteriana dos óleos essenciais de cinco espécies de Eucalyptus cultivadas em Goiás. Rev Bras Farmacogn 11: 95-98.

Farias NMP, Lima EO 2000. Atividade antifúngica de óleos essenciais obtidos de plantas medicinais contra leveduras do gênero Candida: uma alternativa no controle da infecção hospitalar. In: Prêmio Jovem Cientista XVI, Edição Saúde da População, Controle da Infecção Hospitalar. Porto Alegre: Fundação Roberto Marinho, p.91-120.

Gonçalves LA, Barbosa LCA, Azevedo AA, Casali VWD, Nascimento EA 2003. Produção e composição do óleo essencial de alfavaquinha (Ocimim selloi Benth.) em resposta a dois níveis de radiação solar. Revista Brasileira de Plantas Medicinais 6:8-14.

Lima EO 1996. Estudo das dermatofitoses em João PessoaParaíba e da atividade antifúngica de plantas medicinais da região contra alguns de seus agentes isolados. São Paulo, 180p. Tese de Doutorado Faculdade de Ciências Farmacêuticas.

Lima EO, Farias NMP, Souza EL, Santos BHC 2003. Propriedades antibacterianas de óleos essenciais de plantas medicinais. Revista Brasileira de Ciências da Saúde 7: 251-258.

Matos FJA 1988. Introdução à Fitoquímica Experimental. Fortaleza: Editora UFC.

Matos FJA 1994. Farmácias vivas: sistema de utilização de plantas medicinais. Projeto para pequenas comunidades. Fortaleza: Editora UFC.

Menéndez CLRA, González LVP 1999. Plecthranthus amboinicus (Lour.) Spreng. Revista Cubana de Plantas Medicinais 3: 110-115.

Michelin DC, Moreschi PE, Lima, AC, Nascimento GGF, Paganelli MO, Chaud MV 2005. Avaliação da atividade antimicrobiana de extratos vegetais. Rev Bras Farmacogn 15: 316-320.

Nascimento GF, Locatelli J, Freitas PC, Silva GL 2000. Antibacterial activity of plant extracts and phytochemicals on antibiotic-resistant bacteria. Rev Bras Microbiol 31: 48-53.

Nogueira JCR. 2004. Atividade antimicrobiana in vitro de produtos vegetais sobre microrganismos isolados de pacientes portadores de otite externa aguda. João Pessoa, 108p. Dissertação de Mestrado - Programa de Pós-Graduação em Produtos Naturais e Sintéticos Bioativos. Universidade Federal da Paraíba.

Pontes ZBVS 2002. Atividade antifúngica de produtos naturais e sintéticos sobre espécies de Trichosporon behrend. João Pessoa, 178p. Tese de Doutorado - Programa de Pós-graduação em Produtos Naturais e Sintéticos Bioativos, Universidade Federal da Paraíba.

Secretaria de Saúde do Estado da Paraíba 2002. Fitoterápicos: guia do profissional de saúde. Coordenação de Saúde, Núcleo de Assistência Farmacêutica, João Pessoa.

Silva AF, Barbosa LCA, Silva EAM, Casali VWD, Nascimento EA 2003. Composição química do óleo essencial de Hyptis suaveolens (L.) Poit. (Lamiaceae). Revista Brasileira de Plantas Medicinais 6: 1-7.

Simões CMO, Schenkel EP, Gosmann G, Mello JCP, Mentz LA, Petrovick PR 1999. Farmacognosia: da planta ao medicamento. Porto Alegre: Editora UFRS/UFSC.

Siqui AC, Sampaio, ALF, Sousa MC, Henriques MGMO, Ramos, MFS 2000. Óleos essenciais - potencial antiinflamatório. Biotecnologia, Ciência e Desenvolvimento 16: 38-43. 